\title{
Lipid nanoparticles for alkyl lysophospholipid edelfosine encapsulation: development and in vitro characterization
}

Ander Estella-Hermoso de Mendoza ${ }^{1}$, Marta Rayo ${ }^{1}$, Faustino Mollinedo ${ }^{2}$ and María J. Blanco-Prieto $^{1 *}$

${ }^{1}$ Department of Pharmacy and Pharmaceutical Technology, School of Pharmacy, University of Navarra, C/Irunlarrea 1, E-31080 Pamplona, Spain

${ }^{2}$ Centro de Investigación del Cáncer, Instituto de Biología Molecular y Celular del Cáncer, CSIC-Universidad de Salamanca, Campus Miguel de Unamuno, E-37007 Salamanca, Spain

* To whom correspondence should be addressed:

Dr. María J. Blanco Prieto

Department of Pharmacy and Pharmaceutical Technology, School of Pharmacy, University of Navarra, C/Irunlarrea 1, E-31080 Pamplona, Spain

Office phone: + 34948425600 ext. 6519

Fax: + 34948425649

e-mail:mjblanco@unav.es 


\begin{abstract}
The ether lipid 1-O-octadecyl-2-O-methyl-rac-glycero-3-phosphocholine, edelfosine $\left(\mathrm{ET}-18-\mathrm{OCH}_{3}\right)$ is the prototype molecule of a promising class of antitumour drugs named alkyl-lysophospholipid analogues (ALPs) or antitumor ether lipids. This drug presents a very important drawback as can be the dose depending haemolysis when administered intravenously. Lipid nanoparticles have been lately proposed for different drug encapsulation as an alternative to other controlled release delivery systems, such as liposomes or polymeric nanoparticles. The aim of this study was to develop a lipid nanoparticulate system that would decrease systemic toxicity as well as improve the therapeutic potential of the drug. Lipids employed were Compritol ${ }^{\circledR} 888$ ATO and stearic acid. The nanoparticles were characterized by photon correlation spectroscopy for size and size distribution, and atomic force microscopy (AFM) was used for the determination of morphological properties. By both differential scanning calorimetry (DSC) and X-ray diffractometry, crystalline behaviour of lipids and drug was assessed. The drug encapsulation efficiency and the drug release kinetics under in vitro conditions were measured by HPLC-MS. It was concluded that Compritol ${ }^{\circledR}$ presents advantages as a matrix material for the manufacture of the nanoparticles and for the controlled release of edelfosine.
\end{abstract}

Key words: Lipid nanoparticles; Edelfosine; Drug delivery; Atomic force microscopy; Differential scanning calorimetry; X-ray diffractometry. 


\section{Introduction}

Edelfosine

$\left(\mathrm{ET}-18-\mathrm{OCH}_{3}\right.$,

1-O-octadecyl-2-O-methyl-rac-glycero-3phosphocholine) is the prototype of a promising class of antitumor agents, collectively known as alkyl-lysophospholipid analogues (ALPs) or antitumor ether lipids, that do not target the DNA, but affect the cell membrane and the apoptotic machinery of the cancer cell [1]. Phase I studies have shown a good tolerability of the drug but with haematological and systemic side effects $[1,2]$. However, although edelfosine has been shown to exert potent antineoplastic effects in vitro $[3,4]$, the antitumor activity in phase II clinical studies has been only moderate [5]. Moreover, ALPs show manifold biological effects in addition to their antineoplastic actions, including an antiparasitic effect on Leishmania [6] as well as an inhibition of the cell membrane phospholipid turnover [7] and a potent inhibition of neovascularization [8], protein kinase $\mathrm{C}$ and $\mathrm{Na}^{+} / \mathrm{K}^{+}$-ATPase [9].

Edelfosine has also been given intravenously, but this provoked haemolysis as a major side effect [10]. This led to the only formulation developed so far, the TLC ELL12, in which edelfosine was included into liposomes [11] to avoid the haemolytic toxicity of the drug. However, the main inconvenience of liposomes is their rapid clearance from plasma in comparison with other delivery systems.

Lipid nanoparticles have been proposed as an alternative for the existing traditional particulate systems, such as previously mentioned liposomes or polymeric nanoparticles. These particulate systems made from solid lipids started being developed in the early nineties. They provide physical stability, controlled release and a wide variety of application routes (parenteral, oral, dermal, ocular, pulmonary and rectal) [12$16]$. 
Lipid nanoparticles are basically composed of a high melting point lipid that acts as a solid core, covered by surfactants. Lipids used to form these matrices are biodegradable raw materials that are physiologically tolerated [17]: triglycerides (i.e. tristearin), partial glycerides (i.e. Compritol), fatty acids (i.e. stearic acid), steroids (i.e. cholesterol) or waxes (i.e. cetyl palmitate) [18].

The formulation methods are also diverse. Emulsion formation and solvent evaporation method has been widely used for particle formation, although other methods like high pressure homogenization [18], solvent diffusion methods in aqueous solutions [19] or hot emulsion methods [18, 20] have also been employed. Drawbacks associated to this kind of formulations, like limited drug loading capacity, adjustment of drug release profile and potential drug expulsion during storage have been reported [21]. Besides, drug loading capacity is limited by the solubility of the drug in the lipid melt, the structure of the lipid matrix and the polymorphic state of the lipid matrix.

Comparing with the previously mentioned liposomes, the main improvement of the lipid nanoparticles is their physical and chemical long-term stability up to $12-24$ months [13], even though an increase in particle size has been reported in a lesser time [22]. As a feasible solution for this setback, the freeze-drying process has shown to increase physicochemical stability of lipid particles over large periods of time [23].

Taking into account this information, the aim of this study was to develop a new formulation which would provide a controlled release for the antitumor lipid edelfosine, in order to improve its therapeutic activity. 


\section{Materials and Methods}

\subsection{Materials}

Edelfosine was from INKEYSA (Barcelona, Spain). Compritol ${ }^{\circledR} 888$ ATO was a gift of Gattefossè (Cedex, France). Stearic acid and Tween ${ }^{\circledR} 80$ were purchased from Roig Farma (Barcelona, Spain). Poly (vinyl alcohol) $88 \%$ hydrolyzed (MW: 125,000) and Phosphate Buffer Saline (PBS) were provided by Sigma-Aldrich (Spain). Chloroform and ethyl acetate were obtained from Panreac Química S.A. (Barcelona, Spain). All solvents employed for the chromatographic analysis were of analytical grade. Formic acid $99 \%$ for mass spectroscopy was purchased from Fluka (Spain) and methanol was obtained from Merck (Spain). All other chemicals were of reagent grade and used without further purification. Amicon Ultra-15 centrifugal filter devices were purchased from Millipore (Cork, Ireland).

\subsection{Preparation of lipid nanoparticles}

Lipid nanoparticles were prepared by the emulsification/solvent evaporation method. For the simple emulsion solvent evaporation method, edelfosine and the lipid (either Compritol ${ }^{\circledR}$ or stearic acid) were dissolved in chloroform. This solution was emulsified with a $0.5 \%$ or $1 \%$ Tween 80 solution by ultrasonication using a Microson $^{\mathrm{TM}}$ ultrasonic cell disruptor (NY, USA). The $\mathrm{O} / \mathrm{W}$ emulsion formed was magnetically stirred for 45 minutes and subjected to low vacuum rotary evaporation for the complete elimination of the organic solvent. Particles were centrifuged at $4500 \mathrm{~g}$ for 40 minutes using an Amicon Ultra-15 filter device and washed twice with distilled water. The obtained particular suspension was fast frozen under $-80{ }^{\circ} \mathrm{C}$ for at least 3 hours and freeze-dried in order to store it at $4{ }^{\circ} \mathrm{C}$. For the double emulsion $(\mathrm{W} / \mathrm{O} / \mathrm{W})$, edelfosine was dissolved in $100 \mu \mathrm{l}$ of a $1 \%$ Tween 80 solution and emulsified with $1 \mathrm{ml}$ 
of ethyl acetate. This first W/O emulsion was then emulsified with $10 \mathrm{ml}$ of a $1 \%$ Tween 80 solution. Following steps were the same to the ones of the simple emulsion solvent evaporation method.

\subsection{Encapsulation efficiency}

Edelfosine was extracted by dissolving $10 \mathrm{mg}$ of nanoparticles in $1 \mathrm{ml}$ of chloroform and then mixed with $3 \mathrm{ml}$ of ultra pure water. The mixture was vortexed for 1 minute and then centrifuged at $9500 \mathrm{~g}$ for 10 minutes. The supernatant was analysed by a HPLC-MS method, which is a slight modification of a previously developed method [24]. The apparatus used for the HPLC analysis was a Model 1100 series LC coupled with an atmospheric pressure (AP)-electrospray ionization (ESI) mass spectrometer (HP 1100 with MSD VL, Waldbronn, Germany). Data acquisition and analysis were performed with a Hewlett-Packard computer using the ChemStation G2171 AA programme. Separation was carried out at $50{ }^{\circ} \mathrm{C}$ on a reversed-phase, $150 \mathrm{~mm} \times 3 \mathrm{~mm}$

column packed with $\mathrm{C} 18,5 \mu \mathrm{m}$ silica reversed-phase particles $\left(\mathrm{Gemini}^{\circledR}\right)$ obtained from Phenomenex $^{\circledR}$ (Torrance, CA, USA). This column was preceded by a reversed-phase, $\mathrm{C} 18,5 \mu \mathrm{m}$ guard column $\left(\right.$ SecurityGuard $^{\circledR}, 20 \mathrm{~mm} \times 4 \mathrm{~mm}$, Phenomenex ${ }^{\circledR}$, Torrance, CA, USA). The mobile phase was a mixture of methanol-1 \% formic acid $(95: 5, \mathrm{v} / \mathrm{v})$. Separation was achieved by isocratic solvent elution at a flow-rate of $0.5 \mathrm{ml} / \mathrm{min}$. The mass spectrometer was operated in the positive ESI mode. The detection of edelfosine was performed by selected ionization monitoring (SIM) mode. The mass spectrometer was programmed to monitor the ion of edelfosine at $\mathrm{m} / \mathrm{z} 524.40$. Typical retention time was 3.65 minutes. 


\subsection{Nanoparticle characterization}

\subsubsection{Particle size, size distribution and zeta potential}

Particle size and distribution of the nanoparticles were measured by photon correlation spectroscopy (PCS) using a Zetasizer Nano (Malvern Instruments, UK). Each sample was diluted with distilled water until the appropriate concentration of particles was achieved to avoid multiscattering events. The obtained homogenous suspension was examined to determine the volume mean diameter, size distribution and polydispersity.

The electrophoretic mobility and zeta potential were determined by Laser Doppler Velocimetry using a Zetasizer Nano (Malvern Instruments, UK).

\subsubsection{Morphology}

Atomic force microscopy (Cervantes AFM System, Nanotec Electrónica, S.L., Spain) was employed to determine the shape and surface morphology of the nanoparticles. AFM was conducted with Nanoscope IIa IIIa in the tapping mode. The nanoparticle sample was mounted on a metal stab and scanned by the AFM maintained in a constant temperature and vibration time environment.

\subsubsection{Thermal analysis of freeze-dried lipid nanoparticles}

The thermal characteristics of selected batches of nanoparticles were determined by differential scanning calorimetry thermal analysis using a 2920 DSC (Universal V3.6C TA Instruments, USA). The scan rate was $10^{\circ} \mathrm{C} / \mathrm{min}$ in the temperature range from -10 ${ }^{\circ} \mathrm{C}$ to $275{ }^{\circ} \mathrm{C}$ and a $\mathrm{N}_{2}$ flow of $20 \mathrm{~L} / \mathrm{min}$. An empty pan was used as reference standard. Indium (purity $\geq 99.95 \%$, Fluka, Switzerland) was employed to check the calibration of the calorimetric system. 


\subsubsection{X-ray studies of freeze-dried lipid nanoparticles}

X-ray diffraction measurements were performed in order to clearly elucidate the solid state of both lipids and drug in lipid nanoparticles, using a Bruker D8 Advance Xray diffractometer (Bruker Biosciences Española, S.A., Spain). The X-ray diffractogram was scanned with the diffraction angle increasing from $2^{\circ}$ to $40^{\circ}, 2 \theta$ angle, with a step angle of $0.02^{\circ}$ and a count time of $1 \mathrm{~s}$ at a constant temperature of $25^{\circ} \mathrm{C}$.

\subsection{In vitro release studies}

The release rate of edelfosine from lipid nanoparticles was measured in PBS medium ( $\mathrm{pH} 7.4$ ). Briefly, $5 \mathrm{mg}$ of nanoparticles were dispersed in $1 \mathrm{ml}$ of buffer solution and maintained at $37^{\circ} \mathrm{C}$ under stirring (260 rpm). At appropriate time intervals, samples were centrifuged (23500 g, 10 minutes), supernatants were filtrated with a 0.45 $\mu \mathrm{m}$ pore diameter filter and kept at $-20^{\circ} \mathrm{C}$ until further HPLC-MS analysis was conducted as previously described. Three samples were employed for each time and the study was performed in triplicate.

The mean in vitro dissolution time (MDT), a model-independent in vitro parameter that shows the meantime for edelfosine to release from the lipid nanoparticles under in vitro release conditions, was calculated according to the equation:

$$
M D T=\frac{A B C_{\text {in vitro }}}{M_{\infty}}
$$

where $A B C_{\text {in vitro }}$ is the area between the release curve and its asymptote, calculated by the trapezoidal rule from time zero to the last measured time point, and $M_{\infty}$ is the total amount of released drug at this time point. The release rate constant $\left(k_{d}\right)$ was calculated by the expression $k_{d}=1 / \mathrm{MDT}$. 


\section{Results and discussion}

\subsection{Particle size, size distribution and zeta potential}

Structurally, edefosine has a structure of an amphifile, with a part of the molecule exhibiting hydrophobicity and another part exhibiting hydrophilicity, like a surfaceactive agent (Fig.1).

Edelfosine-loaded lipid nanoparticles were obtained by either simple or multiple emulsion solvent evaporation method, and freeze-dried. These nanoparticles were then characterized to assess the effect of the different lipids and surfactant concentrations on mean particle size, size distribution and surface charge.

Using lipids as matrices for the particles, different characteristics can be obtained by optimizing the formulation parameters such as type of lipids, surfactants, organic solvents and emulsifying procedure chosen [18]. The presence of an anionic surfactant is important to reduce the dynamic interfacial tension and to stabilize the nanosuspension. The surfactant is adsorbed on the nanoparticle surface, increasing the steric repulsion between particles. In this study, Tween ${ }^{\circledR} 80$ was tested at two different concentrations (w/v), being sufficient to obtain small lipid nanoparticles and permitting the removal of its excess by centrifugation and washing.

The average size and polydispersity indices of lipid nanoparticles formulated with different lipids are reported in Table 1 . All data are expressed as mean value \pm standard deviation. For all lipids, it was possible to obtain submicron sized lipid nanoparticles with two different concentrations of Tween ${ }^{\circledR} 80$. In fact, all formulations showed a mean diameter in the range of $300-600 \mathrm{~nm}$. There was a decrease in size of particles when formulated with edelfosine (data not shown), probably due to the effect of edelfosine as a surfactant agent. Smallest particles were obtained using stearic acid, chloroform and an aqueous solution of $\mathrm{Tween}^{\circledR} 80$ at a concentration of $1 \%$. The 
polydispersity index (PDI) value was in the range of $0.2-0.6$ for all lipid nanoparticles investigated.

Zeta potential can make a prediction about the stability of colloid dispersions. A high zeta potential $(>30 \mathrm{mV})$ can provide an electric repulsion to avoid the aggregation of particles [25]. The incorporation of edelfosine into lipid nanoparticles had no significant influence on the zeta potentials of particles, which was negative in all cases. However, as the concentration of surfactant was increased in the formulation the zeta potential was found to be more negative (Table 1). This could be due to the adsorption of the surfactant on the particle surface, forming a denser surfactant film, thus eliciting a reduced electrophoretic mobility.

\subsection{Drug entrapment efficiency and loading capacity}

Over the past few years many different drugs had been successfully incorporated in lipid nano- or microparticles [20, 26-28]. Relatively higher drug encapsulation efficiency was one of the major advantages of lipid nanoparticles. It is also known that the lipid crystalline structure related to the chemical nature of the lipid is a key factor to determine whether a drug will be expelled or firmly incorporated into the carrier systems. In the nanoparticle structure, the lipid forming highly crystalline state with a perfect lattice would lead to drug expulsion. On the other hand, imperfections (lattice defects) of the lipid structure could offer space to accommodate the drugs [29]. As a result, the structure of less ordered arrangement in the nanoparticles would be beneficial to the drug loading capacity like the samples in this study.

From the results listed in Table 2, it can be observed that the entrapment efficiency of edelfosine in the lipid nanoparticles prepared by the simple emulsion solvent evaporation method ranged from about 4 to $10 \%$ for stearic acid nanoparticles. On the 
other hand, nanoparticles formulated using Compritol ${ }^{\circledR}$ encapsulated more than $80 \%$ of the drug (Table 2). This high encapsulation efficiency in comparison to the stearic acid nanoparticles is likely to be due to the partially amorphous state of the Compritol $^{\circledR}$ in the formulation, which allows more edelfosine to be incorporated among lipid chains. It can also be observed that the emulsifier concentrations investigated in this study $(0.5 \%$ and $1 \%$ Tween 80 ) do not affect the encapsulation efficiency.

On the other hand, lipid nanoparticles prepared by the multiple emulsion solvent evaporation method showed very low encapsulation efficiency, less than $1 \%$ (data not shown).

\subsection{Morphology}

In order to investigate the shape and surface morphology of the Compritol ${ }^{\circledR}$ nanoparticles, atomic force microscopy was employed. The AFM images reveal the fine structure of the Compritol ${ }^{\circledR}$ lipid nanoparticle surface (Fig. 2A). They give clear 3D morphological images of spherical nanoparticles of sub-400 $\mathrm{nm}$ diameter and they also confirm that there was no aggregation or adhesion among the nanoparticles (Fig. 2C). Furthermore, the surface morphology of the nanoparticles could be seen closely from the AFM images. It was noticeable from the zoom-in picture (Fig. 2B) the smooth surface morphology of the nanoparticles.

\subsection{DSC and X-ray diffractometry assays}

Lipid nanoparticles were analysed by DSC and X-ray diffractometry to investigate the crystal pattern of both edelfosine and lipids, because this aspect could influence the in vitro and in vivo release of the drug from the systems. To probe this effect, analysis were performed on the following samples: edelfosine; Compritol ${ }^{\circledR} 888$ ATO; stearic 
acid; edelfosine loaded stearic acid nanoparticles; edelfosine loaded Compritol ${ }^{\circledR}$ nanoparticles; unloaded stearic acid nanoparticles; unloaded Compritol ${ }^{\circledR}$ nanoparticles.

Fig. 3 depicts the DSC thermograms of edelfosine loaded and unloaded lyophilised nanoparticles. DSC analysis of edelfosine-loaded lipid nanoparticles showed that the drug melting peak at $223^{\circ} \mathrm{C}$ is present neither in the stearic acid lipid nanoparticles nor in the Compritol ${ }^{\circledR}$ lipid nanoparticles whereas for the pure drug, the melting peak occurs before its decomposition. This thermal behaviour may be ascribed to the presence of edelfosine in an amorphous form or molecularly dispersed. This effect on the crystalline habit of edelfosine may be related to the preparative method of the lipid nanoparticles, in which edelfosine may be turned from a crystalline state to an amorphous one by the use of organic solvents like chloroform or methylene chloride.

Thermal behaviour of lipids can also explain the different encapsulation efficiency of edelfosine. Unloaded stearic acid nanoparticles showed the melting peak of the stearic acid at $59{ }^{\circ} \mathrm{C}$, indicating its presence in crystalline state, thus letting less amount of drug to be incorporated among its lipidic chains. On the other hand, Compritol ${ }^{\circledR}$ nanoparticles seem to lose part of their crystalline state, permitting edelfosine to fit in the molecular gaps. These findings were confirmed by X-ray diffractometry assays. Unloaded stearic acid nanoparticles (Fig. 4A) show two sharp peaks corresponding to those of the stearic acid, whereas the diffraction pattern of Compritol ${ }^{\circledR}$ (Fig. 4B) showed a typical double peak that is significantly modified when formulated into nanoparticles. Comparing these results with edelfosine loaded lipid nanoparticles, some crystalline drug signal could still be detected, indicating a possible coexistence of edelfosine in both crystalline and amorphous states, being this last one the predominant. As a result, edelfosine would enrich the particle surface when formulated with stearic acid, whereas it would be incorporated among the lipid chains of Compritol ${ }^{\circledR}$. 


\subsection{In vitro release studies}

The amount of edelfosine released from lipid nanoparticles was determined by an in vitro release assay, in an effort to assess whether edelfosine-incorporating lipid nanoparticles might be useful as a sustained-release dosage form.

Fig. 5 displays the release profiles for nanoparticles fabricated with stearic acid or Compritol $^{\circledR}$ using $1 \%$ Tween 80 as emulsifier. When edelfosine was incorporated in stearic acid nanoparticles, a $63 \%$ burst release of the drug was observed within 20 minutes (Fig.5). Conversely, the initial release burst for nanoparticles prepared using Compritol $^{\circledR}$ was less than $40 \%$ within the first 20 minutes. The reason for the high initial release of edelfosine from the stearic acid nanoparticles could be the diffusion release of edelfosine distributed near the surface and in the outer portion of the nanoparticles [12]. These results were in accordance with the observations made by DSC and X-ray diffractometry.

Afterwards, the release rate slowed for both formulations, reaching $55 \%$ after $24 \mathrm{~h}$ for nanoparticles prepared with Compritol $^{\circledR}$, and $98 \%$ for the stearic acid nanoparticles.

The release of a drug from the dosage form implies a crucial step, which is the dissolution of the drug. This process is ruled by a release rate constant $\left(k_{d}\right)$ that can be easily estimated and it is different depending, among other factors, on the composition of the particle. In our study, this constant was estimated for the release of edelfosine from lipid nanoparticles formulated either with stearic acid or Compritol ${ }^{\circledR}$.

Results showed that there is a different release profile depending on the lipid. Stearic acid presents a higher release rate constant $\left(k_{d}=0.208\right)$ than $\operatorname{Compritol}^{\circledR}\left(k_{d}=\right.$ 0.178), indicative of a faster release. Besides, there seems to be no influence of the amounts of surfactant employed in the release kinetics of the drug, since the release rate 
constant from nanoparticles prepared with different surfactant concentrations $(0.5$ or $1 \%$ Tween 80) were equivalent. 


\section{Conclusions}

The present research paper proposed a novel formulation for edelfosine by using lipid nanoparticles. It can be concluded that Compritol $^{\circledR}$ presents advantages as a matrix material for the manufacture of the nanoparticles and the controlled release of edelfosine.

Current studies are aimed at evaluating the in vitro (in cell lines) and in vivo efficacy of these newly developed formulations. 


\section{Acknowledgements}

This work was supported by "Fundación Caja Navarra". The authors are grateful to Nanotec Electrónica S.L. and Dr. Blanca Galar for her assistance in X-Ray diffraction studies. The first author acknowledges a fellowship from the Department of Education of the Basque Government. 


\section{References}

[1] C. Gajate, F. Mollinedo, Biological activities, mechanisms of action and biomedical prospect of the antitumor ether phospholipid ET-18-OCH3 (edelfosine), a proapoptotic agent in tumor cells. Curr. Drug. Metab. 3(5) (2002) 491-525.

[2] W.E. Berdel, U. Fink, J. Rastetter, Clinical phase I pilot study of the alkyl lysophospholipid derivative ET-18-OCH3. Lipids 22(11) (1987) 967-969.

[3] F. Mollinedo, J.L. Fernandez-Luna, C. Gajate, B. Martin-Martin, A. Benito, R. Martinez-Dalmau, M. Modolell, Selective induction of apoptosis in cancer cells by the ether lipid ET-18- $\mathrm{OCH}_{3}$ (Edelfosine): molecular structure requirements, cellular uptake, and protection by Bcl-2 and Bcl-X(L). Cancer Res. 57(7) (1997) $1320-1328$.

[4] C. Gajate, F. Mollinedo, Edelfosine and perifosine induce selective apoptosis in multiple myeloma by recruitment of death receptors and downstream signaling molecules into lipid rafts. Blood 109(2) (2007) 711-719.

[5] F. Mollinedo, Antitumour ether lipids: proapoptotic agents with multiple therapeutic indications. Expert Opinion on Therapeutic Patents (In Press) (2007).

[6] S. Azzouz, M. Maache, R.G. Garcia, A. Osuna, Leishmanicidal activity of edelfosine, miltefosine and ilmofosine. Basic Clin. Pharmacol. Toxicol. 96(1) (2005) 60-65.

[7] K.P. Boggs, C.O. Rock, S. Jackowski, Lysophosphatidylcholine and 1-Ooctadecyl-2-O-methyl-rac-glycero-3-phosphocholine inhibit the CDP-choline pathway of phosphatidylcholine synthesis at the CTP:phosphocholine cytidylyltransferase step. J. Biol. Chem. 270(13) (1995) 7757-7764. 
[8] W.R. Vogler, J. Liu, O. Volpert, E.W. Ades, N. Bouck, The anticancer drug edelfosine is a potent inhibitor of neovascularization in vivo. Cancer Invest. 16(8) (1998) 549-553.

[9] B. Zheng, K. Oishi, M. Shoji, H. Eibl, W.E. Berdel, J. Hajdu, W.R. Vogler, J.F. Kuo, Inhibition of protein kinase C, (sodium plus potassium)-activated adenosine triphosphatase, and sodium pump by synthetic phospholipid analogues. Cancer Res. 50(10) (1990) 3025-3031.

[10] I. Ahmad, J.J. Filep, J.C. Franklin, A.S. Janoff, G.R. Masters, J. Pattassery, A. Peters, J.J. Schupsky, Y. Zha, E. Mayhew, Enhanced therapeutic effects of liposome-associated 1-O-octadecyl-2-O-methyl-sn-glycero-3-phosphocholine. Cancer Res. 57(10) (1997) 1915-1921.

[11] R. Bhamra, L.E. Bolcsak, I. Ahmad, J. Schupsky, P. Roberts, R. Stevens, C. Cavanaugh, C.E. Swenson, Activity, pharmacokinetics and tissue distribution of TLC ELL-12 (liposomal antitumor ether lipid) in rats with transplantable, s.c. methylnitrosourea-induced tumors. Anticancer Drugs 14(6) (2003) 481-486.

[12] A. zur Muhlen, C. Schwarz, W. Mehnert, Solid lipid nanoparticles (SLN) for controlled drug delivery--drug release and release mechanism. Eur. J. Pharm. Biopharm. 45(2) (1998) 149-155.

[13] B. Siekmann, K. Westesen, Sub-micron sized parenteral carrier systems based on solid lipids. Pharm. Pharmacol. Lett. 1 (1992) 123.

[14] C. Schwarz, W. Mehnert, Solid lipid nanoparticles (SLN) for controlled drug delivery. II. Drug incorporation and physicochemical characterization. J. Microencapsul. 16(2) (1999) 205-213.

[15] T. de Vringer, H.A. de Ronde, Preparation and structure of a water-in-oil cream containing lipid nanoparticles. J. Pharm. Sci. 84(4) (1995) 466-472. 
[16] A.J. Almeida, S. Runge, R.H. Muller, Peptide-loaded solid lipid nanoparticles (SLN): Influence of production parameters. Int. J. Pharm. 149(2) (1997) 255265.

[17] S.A. Wissing, O. Kayser, R.H. Muller, Solid lipid nanoparticles for parenteral drug delivery. Adv. Drug Deliv. Rev. 56(9) (2004) 1257-1272.

[18] W. Mehnert, K. Mader, Solid lipid nanoparticles: production, characterization and applications. Adv. Drug Deliv. Rev. 47(2-3) (2001) 165-196.

[19] F.Q. Hu, S.P. Jiang, Y.Z. Du, H. Yuan, Y.Q. Ye, S. Zeng, Preparation and characterization of stearic acid nanostructured lipid carriers by solvent diffusion method in an aqueous system. Colloids Surf. B Biointerfaces 45(3-4) (2005) 167-173.

[20] S. Jaspart, P. Bertholet, G. Piel, J.M. Dogne, L. Delattre, B. Evrard, Solid lipid microparticles as a sustained release system for pulmonary drug delivery. Eur. J. Pharm. Biopharm. 65(1) (2007) 47-56.

[21] R.H. Muller, M. Radtke, S.A. Wissing, Nanostructured lipid matrices for improved microencapsulation of drugs. Int. J. Pharm. 242(1-2) (2002) 121-128.

[22] C. Freitas, R.H. Muller, Effect of light and temperature on zeta potential and physical stability in solid lipid nanoparticles (SLN) dispersion. Int. J. Pharm. 168 (1998) 221-229.

[23] R. Cavalli, O. Caputo, M.E. Carlotti, M. Trotta, C. Scarnecchia, M.R. Gasco, Sterilization and freeze -drying of drug-free and drug-loaded solid lipid nanoparticles. Int. J. Pharm. 148 (1997) 47-54.

[24] M.J. Blanco-Prieto, M.A. Campanero, F. Mollinedo, Quantitative determination of the antitumor alkyl ether phospholipid edelfosine by reversed-phase liquid chromatography-electrospray mass spectrometry: application to cell uptake 
studies and characterization of drug delivery systems. J. Chromatogr. B Analyt. Technol. Biomed. Life Sci. 810(1) (2004) 85-92.

[25] M.Y. Levy, W. Schutze, C. Fuhrer, S. Benita, Characterization of diazepam submicron emulsion interface: role of oleic acid. J. Microencapsul. 11(1) (1994) 79-92.

[26] H. Chen, X. Chang, D. Du, W. Liu, J. Liu, T. Weng, Y. Yang, H. Xu, X. Yang, Podophyllotoxin-loaded solid lipid nanoparticles for epidermal targeting. J. Control. Release 110(2) (2006) 296-306.

[27] F.Q. Hu, H. Yuan, H.H. Zhang, M. Fang, Preparation of solid lipid nanoparticles with clobetasol propionate by a novel solvent diffusion method in aqueous system and physicochemical characterization. Int. J. Pharm. 239(1-2) (2002) 121-128.

[28] M.K. Lee, S.J. Lim, C.K. Kim, Preparation, characterization and in vitro cytotoxicity of paclitaxel-loaded sterically stabilized solid lipid nanoparticles. Biomaterials 28(12) (2007) 2137-2146.

[29] D. Hou, C. Xie, K. Huang, C. Zhu, The production and characteristics of solid lipid nanoparticles (SLNs). Biomaterials 24(10) (2003) 1781-1785. 